\title{
STABILITY, INSTABILITY, OSCILLATION AND NONOSCILLATION IN SCALAR INTEGRODIFFERENTIAL SYSTEMS
}

\author{
K. Gopalsamy \\ Sufficient conditions are obtained for a homogeneous scalar \\ integrodifferential system to have solutions all of which either \\ converge to zero or oscillate or at least one of which can be \\ nonoscillatory asymptotically.
}

\section{Introduction}

The purpose of this article is to derive a set of sufficient conditions for scalar integrodifferential equations of the form

$$
\frac{d x(t)}{d t}=a x(t)+b \int_{-\infty}^{t} k(t-s) x(s) d s, t>0,
$$

to have solutions (when supplemented with suitable initial conditions) which converge to zero as $t \rightarrow \infty$, oscillate or do not oscillate asymptotically. Equations of the form (1.1) can be regarded as differential equations with an unbounded delay; for a survey on equations with unbounded delays we refer to Corduneanu and Lakshmikantham [4].

The following lemma establishes an a priori estimate for solutions of equations of the form (1.1) and such an estimate will also justify the application of Laplace transform techniques to systems such as (1.1).

LEMMA 1.1. Suppose $a$ and $b$ are real constants and let

Received I July 1983.

Copyright Clearance Centre, Inc. Serial-fee code: 0004-9727/83 $\$ A 2.00+0.00$. 
$k:[0, \infty) \rightarrow(-\infty, \infty)$ be integrable such that

$$
\int_{0}^{\infty}|k(s)| d s<\infty, \quad \int_{0}^{\infty} s|k(s)| d s<\infty .
$$

Let $\phi:(-\infty, 0] \rightarrow(-\infty, \infty)$ be any bounded function. Then any solution of the initial value problem

$$
\frac{d x(t)}{d t}=a x(t)+b \int_{c}^{t} k(t-s) x(s) d s, t>0,
$$

$$
x(s)=\phi(s), \quad s \in(c, 0],
$$

where $c=-\infty$ or $c=0$ satisfies an estimate of the form

(1.4) $|x(t)|$

$$
\leq\left[|\phi(0)|+b\|\phi\| \int_{0}^{\infty} s|k(s)| d s\right] \exp \left[\left(|a|+|b| \int_{0}^{\infty}|k(s)| d s\right) t\right], t \geq 0,
$$

where $\|\phi\|=\sup _{s \in(c, 0]}|\phi(s)|$.

Proof. The local existence of solutions of (1.2)-(1.3) can be discussed by the usual methods (see for instance Miller [8]). The global existence (for all $t \geq 0$ ) of solutions of (1.2)-(1.3) will be a consequence of our lemma. We will consider only the case $c=-\infty$ and the proof for $c=0$ is similar. We can rewrite (1.3) in the form

$$
\begin{aligned}
\frac{d x}{d t} & =a x(t)+b \int_{-\infty}^{0} k(t-s) \phi(s) d s+b \int_{0}^{t} k(t-s) x(s) d s \\
& \leq a x(t)+|b| \| \phi|| \int_{t}^{\infty}|k(s)| d s+b \int_{0}^{t} k(t-s) x(s) d s .
\end{aligned}
$$

Hence from (1.5),

$$
\text { (1.6) } \begin{aligned}
x(t)-x(0) \leq a \int_{0}^{t} x(s) d s+|b||| \phi \mid & \int_{0}^{t}\left\{\int_{s}^{\infty}|k(u)| d u\right\} d s \\
& +b \int_{0}^{t}\left\{\int_{0}^{s}|k(s-v)||x(v)| d v\right\} d s .
\end{aligned}
$$

Simplifying (1.6), 


$$
\begin{aligned}
& |x(t)| \leq|\phi(0)|+|a| \int_{0}^{t}|x(s)| d s+|b||\phi|\left|\int_{0}^{\infty} s\right| k(s) \mid d s \\
& +b \int_{0}^{t}|x(s)| d s\left(\int_{0}^{\infty}|k(u)| d u\right)
\end{aligned}
$$

that is,

(1.7) $|x(t)| \leq\left(|\phi(0)|+|b|\|\phi\| \int_{0}^{\infty} s|k(s)| d s\right)$

$$
+\left(|a|+|b| \int_{0}^{\infty}|k(s)| d s\right) \int_{0}^{t}|x(u)| d u .
$$

The result follows from (1.7) by an application of Gronwall's lema (Braver and Nohel [2]).

The result of Lemma 1.1 provides sufficient conditions (a priori verifiable) for the solution of (1.3) to be of exponential order; in fact (1.4) provides an a priori estimate for the solution of (1.2)-(1.3). We note that an estimate of the type (1.4) is also valid for scalar systems of the form

$$
\frac{d u(t)}{d t}=a u(t)+\sum_{j=1}^{n} b_{j} \int_{c}^{t} k_{j}(t-s) u(s) d s, c=-\infty \text { or } 0,
$$

$$
u(s)=\phi(s), s \in(c, 0] \text {, }
$$

where $a, b_{1}, \ldots, b_{n}$ are real constants and $\phi, k_{1}, \ldots, k_{n}$ are of the type in Lemma 1.1. The following result provides a set of sufficient conditions for the asymptotic stability and instability of the trivial solution of (1.8); if all solutions of (1.8) exist for all $t \geq 0$ and converge to zero as $t \rightarrow \infty$ then the trivial solution of (1.8) is said to be asymptotically stable and otherwise the trivial solution is said to be "unstable". The following result provides a set of sufficient conditions for the asymptotic stability and instability of the trivial solution of $(1.8)$.

THEOREM 1.1. Sippose the delay kermels $k_{j}(j=1,2, \ldots, n)$ of (1.8) are piecewise continuous such that 


$$
\begin{array}{r}
\text { (1.9) } k_{j}:[0, \infty) \rightarrow(-\infty, \infty), \int_{0}^{\infty} s\left|k_{j}(s)\right| d s<\infty, \int_{0}^{\infty}\left|k_{j}(s)\right| d s<\infty, \\
j=1,2, \ldots, n .
\end{array}
$$

Then every solution of (1.8)-(1.9) comesponding to bounded initial conditions $\phi:(-\infty, 0] \rightarrow(-\infty, \infty)$ converges to zero as $t \rightarrow \infty$ provided the real constants $a, b_{1}, \ldots, b_{n}$ satisfy

$$
a<0,|a|>\sum_{j=1}^{n} b_{j}\left\|k_{j}\right\|,\left\|k_{j}\right\|=\int_{0}^{\infty}\left|k_{j}(s)\right| d s .
$$

If instead of (1.10) we have

$$
a+\sum_{j=1}^{n} b_{j} \int_{0}^{\infty}\left|k_{j}(s)\right| d s \geq 0
$$

then (1.8)-(1.9) will have at least one solution which does not converge to zero as $t \rightarrow \infty$.

Proof. Suppose (1.10) holds and let $c=-\infty$ in (1.8). Consider a Lyapunov function $v(t) \equiv V(t, u)$ where

(1.12) $v(t) \equiv V(t, u)=|u(t)|+\sum_{j=1}^{n}\left|b_{j}\right| \int_{0}^{\infty}\left|k_{j}(s)\right|\left\{\int_{t-s}^{t}|u(n)| d n\right\} d s$.

One can verify that (1.9) and the boundedness of $\phi$ imply that

$$
v(0) \leq|\phi(0)|+\|\phi\| \sum_{j=1}^{n}\left|b_{j}\right| \int_{0}^{\infty} s\left|k_{j}(s)\right| d s<\infty .
$$

Computing the upper right Dini-derivative of $v$ along the solutions of (1.8) (with $c=-\infty$ ) and simplifying we derive that

$$
\begin{aligned}
D^{+} v(t) & \leq-\left\{|a|-\sum_{j=1}^{n}\left|b_{j}\right|\left\|k_{j}\right\|\right\}|u(t)| \\
& \leq-\delta|u(t)|
\end{aligned}
$$

where $\delta=|a|-\sum_{j=1}^{n}\left|b_{j}\right|\left\|k_{j}\right\|>0$. It follows that

$$
v(t)-v(0) \leq-\delta \int_{0}^{t}|u(s)| d s
$$


or since $|u(t)| \leq v(t)$ we have

$$
|u(t)|+\delta \int_{0}^{t}|u(s)| d s \leq v(0)<\infty, t>0 .
$$

(1.15) implies that $u \in L_{1}(0, \infty)$ and $u(t)$ is bounded for $t \geq 0$. Since the right side of (1.8) is bounded under (1.9) for bounded $u$, it will follow that $u$ is uniformly continuous on $[0, \infty)$; such a uniform continuity along with the fact $u \in L_{1}(0, \infty)$ implies that $u(t) \rightarrow 0$ as $t \rightarrow \infty$.

The proof for the case $c=0$ is similar; we rewrite (1.8) as follows :

$$
\frac{d u}{d t}=a u(t)+\sum_{j=1}^{n} b_{j} \int_{-\infty}^{t} k_{j}(t-s) u(s) d s-f(t), t>0,
$$

where

$$
f(t)=\sum_{j=1}^{n} b_{j} \int_{-\infty}^{0} k_{j}(t-s) \phi(s) d s
$$

One can see that

$$
|f(t)| \leq\|\phi\| \sum_{j=1}^{n}\left|b_{j}\right| \int_{t}^{\infty}\left|k_{j}(n)\right| d n
$$

and hence

$$
\begin{aligned}
\int_{0}^{t}|f(s)| d s & \leq\|\phi\| \sum_{j=1}^{n}\left|b_{j}\right| \int_{0}^{t}\left\{\int_{s}^{\infty}\left|k_{j}(n)\right| d n\right\} d s \\
& \leq\|\phi\| \sum_{j=1}^{n}\left|b_{j}\right| \int_{0}^{\infty} n\left|k_{j}(n)\right| d n
\end{aligned}
$$

implying that $f \in L_{1}(0, \infty)$. Thus for the case $c=0$ in (1.8), we can use $(1.16)-(1.19)$. The Lyapunor function $v$ in (1.12) will in this case lead to

$$
D^{+} v(t) \leq-\left[|a|-\sum_{j=1}^{n}\left|b_{j}\right|\left\|k_{j}\right\|\right]|u(t)|+|f(t)| .
$$

Since $f \in L_{1}(0, \infty)$, it will again follow from (1.20) as before that 
$u(t) \rightarrow 0$ as $t \rightarrow \infty$.

We will supply the remainder of the proof assuming that $c=-\infty$ in (1.8) and the modifications needed for the case $c=0$ are minimal. Since the solutions of (1.8)-(1.9) are of exponential order (Lemma 1.1) one can show by means of Laplace transform techniques that if $\tilde{u}(z)$ denotes the Laplace transform of $u$ then we will have

$$
\tilde{u}(z)=(\phi(0+)+F(z)) /\left(z-\left\{a+\sum_{j=1}^{n} b_{j} \int_{0}^{\infty} e^{\left.-z s_{k}(s) d s\right\}}\right)\right.
$$

where

$$
F(z)=\int_{0}^{\infty} e^{-z v}\left\{\int_{-\infty}^{0} k(v-s) \phi(s) d s\right\} d v
$$

Now we consider the solution corresponding to $\phi(s)=0, s \in(-\infty, 0)$, $\phi(0+) \neq 0$. For such a solution we have

$$
\tilde{u}(z)=\phi(0+) /\left(z-\left\{a+\sum_{j=1}^{n} b_{j} \int_{0}^{\infty} e^{-z s_{k}}(s) d s\right\}\right) .
$$

It is known from the theory of Laplace transforms (see Churchill [3]) that corresponding to each simple zero $\alpha$ of

$$
g(z)=z-\left\{a+\sum_{j=1}^{n} b_{j} \int_{0}^{\infty} e^{-z s} k_{j}(s) d s\right\}
$$

there exists a solution $u(t)$ in the form $u(t)=A e^{\alpha t}$ for some constant $A$ (not necessarily real). Thus it will follow from

$$
a+\sum_{j=1}^{n} b_{j} \int_{0}^{\infty} k_{j}(s) d s=0
$$

that $g$ in (1.23) has a zero given by $z=0$ corresponding to which there is a solution $u(t) \equiv$ a constant. If however

$$
a+\sum_{j=1}^{n} b_{j} \int_{0}^{\infty} k_{j}(s) d s>0
$$

then $g$ in (1.23) has a real positive zero say $B>0$ corresponding to which we have a solution of the form say $u(t)=A e^{\beta t}$ for some constant $A$. To see the existence of such a positive zero of $g$ under (1.25) we 
let

$$
\begin{aligned}
& f_{1}(z)=z, \\
& f_{2}(z)=a+\sum_{j=1}^{n} b_{j} \int_{0}^{\infty} k_{j}(s) e^{-z s} d s,
\end{aligned}
$$

and plot the graphs of $f_{1}$ and $f_{2}$ for $z \geq 0$. Since $f_{2}(0)>0$, $f_{2}(z) \rightarrow a<0$ as $z \rightarrow \infty$ and $f_{2}$ is monotonically decreasing in $z$ for $z>0$ it will follow that there exists a unique positive number say $\beta$ such that

$$
f_{1}(\beta)=f_{2}(\beta)
$$

The proof is now complete.

\section{Oscillatory and nonoscillatory solutions}

We shall now derive a set of sufficient conditions on the parameters of a scalar integrodifferential system so that all solutions of the system oscillate or the system has at least one nonoscillatory solution. A nonconstant solution defined on $(-\infty, \infty)$ is said to be oscillatory if it has arbitrarily large zeros on $[0, \infty)$; otherwise a nonconstant solution is said to be nonoscillatory. We can now formulate the following.

THEOREM 2.1. Consider the system

$$
\frac{d y}{d t}=a y(t)-b \int_{c}^{t} k(t-s) y(s) d s, t>0,
$$

$$
y(s)=\phi(s), \quad s \in(-\infty, 0], \phi(0+) \neq 0,
$$

where $c=-\infty$ or $c=0$;

(2.2) $k:[0, \infty) \rightarrow[0, \infty), 0<\int_{0}^{\infty} k(s) d s<\infty, \int_{0}^{\infty} s k(s) d s<\infty$,

$a$ being cony real constant while $b$ is a positive constant. Then $a$ sufficient condition for all solutions of (2.1)-(2.2) corresponding to bounded initial conditions $\phi$ on $(-\infty, 0]$ to be oscillatory is

$$
e b \int_{0}^{\infty} k(s) e^{-a s} s d s>1
$$


If (2.3) fails to hold then (2.1)-(2.2) can have nonoscillatory solutions.

Proof. First suppose $c=-\infty$ and let

$$
y(t)=u(t) e^{a t}
$$

in (2.1). Then the oscillatory or nonoscillatory nature of $y$ will be governed by that of $u$; we derive from (2.1) and (2.4) that

$$
\frac{d u}{d t}=-b \int_{0}^{\infty} k(s) e^{-a s} u(t-s) d s, t>0 .
$$

It will now follow from Lemma 1.1 that the Laplace transform of $y$ in (2.1) exists and hence that of $u$ will exist. If $\tilde{u}(z)$ denotes the Laplace transform of $u$ in (2.5) then it can be found that

$$
\tilde{u}(z)=(\phi(0+)+\Phi(z)) /\left(z+b \int_{0}^{\infty} k(s) e^{-a s} e^{-z s} d s\right)
$$

where $\Phi(z)$ denotes the transform of

$$
-b \int_{t}^{\infty} k(s) e^{-a s} u(t-s) d s
$$

which is related to the initial condition $\phi$ and hence $\Phi$ is a known entity. From the convolution theorem on Laplace transforms (Churchill [3]),

$$
u(t)=\phi(0+) U(t)+\int_{0}^{t} U(t-s)\left\{-\int_{s}^{\infty} k(n) e^{-a n} u(s-n) d n\right\} d s
$$

where $U(t)$ denotes the inverse Laplace transform of

$$
1 /\left(z+b \int_{0}^{\infty} k(s) e^{-a s} e^{-z s} d s\right) \text {. }
$$

It follows from (2.7) that $u(t)$ will be oscillatory or nonoscillatory for all initial bounded conditions if and only if $U(t)$ is oscillatory or nonoscillatory; from the inversion theorem on Laplace transforms we note that $U(t)$ will be oscillatory if and only if all the roots of

$$
z=-b \int_{0}^{\infty} k(s) e^{-a s} e^{-z s} d s
$$


have nonzero imaginary parts; that is $(2.8)$ has no purely real roots.

It is clear that $z=0$ cannot be a root of (2.8); since $b>0$, $k(s) \geq 0$ on $[0, \infty),(2.8)$ cannot have a real positive root also. We will show that when (2.3) holds, (2.8) cannot have a real negative root. We let first $\mu=-z$ in (2.8) and rewrite (2.8) in the form

$$
\begin{aligned}
I & =b \int_{0}^{\infty} k(s) e^{-a s}\left(\frac{e^{\mu s}}{\mu}\right) d s, \mu=-z, \\
& =h(\mu) \quad \text { (say) }
\end{aligned}
$$

and consider the nature of $h(\mu)$ for $\mu>0$. From elementary considerations one finds that $h(\mu)$ for $\mu>0$ is bounded below such that

$$
\inf _{\mu \geq 0} h(\mu)=e b \int_{0}^{\infty} k(s) e^{-a s} s d s .
$$

Thus (2.3), (2.8), (2.9) and (2.10) imply that (2.8) cannot have a real negative root. Already we have verified that (2.8) cannot have a nonnegative root. Thus (2.8) has no real roots and hence all the solutions of (2.1)-(2.2) will be oscillatory when (2.3) holds. In the case corresponding to $c=0$ we will not have $\Phi$ in (2.6) and other details are identical.

If (2.3) fails to hold, (2.8) can have real roots (as seen from (2.9)-(2.10)) corresponding to which (2.1)-(2.2) can have nonoscillatory solutions and the proof is complete.

The following corollaries are easy consequences of Theorem 2.1 and hence we will suppress their proofs.

COROLLARY 2.1. Let $a, b, \tau$ be real constants such that $b>0$, $\tau>0$. Then a sufficient condition for all solutions of

$$
\frac{d y}{d t}=a y(t)-b y(t-\tau)
$$

to be oscizzatory is

$$
\text { bere } e^{-a \tau}>1 \text {. }
$$

COROLLARY 2.2. Let $a$ be a real constant and let $b_{1}, b_{2}, \ldots, b_{n}$ be positive constants; let $k_{j}:[0, \infty) \rightarrow[0, \infty)$ be such that 


$$
\int_{0}^{\infty} k_{j}(s) d s<\infty, \int_{0}^{\infty} s k_{j}(s) d s<\infty, j=1,2, \ldots, n .
$$

Then a sufficient condition for all solutions of

$$
\frac{d y}{d t}=a y-\sum_{j=1}^{n} b_{j} \int_{c}^{t} k_{j}(t-s) y(s) d s, t>0, c=-\infty \text { or } c=0,
$$

corresponding to bounded initial conditions on $(-\infty, 0]$ to be oscillatory is that

$$
e \sum_{j=1}^{n} b_{j} \int_{0}^{\infty} k_{j}(s) e^{-a s} s d s>1
$$

or

$$
n e\left\{\prod_{i=1}^{n}\left(b_{j} \int_{0}^{\infty} k_{j}(s) s e^{-a s} d s\right)\right\}^{1 / n}>1
$$

\section{Equations with variable coefficients}

We shall briefly consider a scalar integrodifferential system of the form

$$
\begin{aligned}
& \frac{d x(t)}{d t}=a(t) x(t)-\int_{0}^{t} k(t, s) x(s) d s, t>0, \\
& x(0)=x_{0} \neq 0,
\end{aligned}
$$

where $a$ is continuous on $[0, \infty)$ and $k(t, s) \geq 0$, continuous for $0 \leq s \leq t<\infty$ such that $k(t, s)>0$ for some $s \geq 0$. We can simplify (3.1) by letting

$$
x(t)=y(t) \exp \left[\int_{0}^{t} a(s) d s\right]
$$

so that

$$
\frac{d y(t)}{d t}=-\int_{0}^{t} k(t, s) \exp \left[-\int_{s}^{t} a(u) d u\right] y(s) d s .
$$

If we consider solutions of (3.3) in the form 


$$
y(t)=\exp \left[-\int_{0}^{t} \lambda(\gamma) d \gamma\right]
$$

for not necessarily real valued functions $\lambda(t)$ defined for $t \geq 0$ then it will follow that $y$ and hence $x$ will be oscillatory if and only if there is no real valued function $\lambda:[0, \infty) \rightarrow(-\infty, \infty)$; it is found that $\lambda(\cdot)$ is governed by

$$
\lambda(t)=\int_{0}^{t} k(t, s) \exp \left[-\int_{s}^{t} a(u) d u\right] \exp \left[\int_{s}^{t} \lambda(\gamma) d \gamma\right] d s .
$$

It is also found from (3.5) that $\lambda(\cdot)$ cannot be negative and $\lambda(t)$ 丰 0 . We formulate our result in the following.

THEOREM 3.1. Assume that $a(t)$ and $k(t, s)$ are continuous respectively for $t \geq 0$ and $0 \leq s \leq t<\infty$. Suppose further that

$$
k(t, s) \geq 0,0 \leq s \leq t<\infty \text { and } k(t, s)>0 \text { for some } s \geq 0 \text {. }
$$

If

$$
\underset{t \rightarrow \infty}{\lim \sup }\left[e \int_{0}^{t}\left\{\int_{0}^{v} k(v, s) \exp \left[-\int_{s}^{v} a(u) d u\right] d s\right\} d v\right]>1
$$

then every solution of (3.1) is oscillatory. If (3.6) fails to hold, (3.1) can have nonoscizlatory solutions.

Proof. It is enough to prove from the foregoing preparation that there exists no real valued $\lambda$ satisfying (3.5). We will prove the nonexistence of real $\lambda$ when (3.6) holds as follows. Suppose (3.6) holds and a real $\lambda$ exists satisfying (3.5). Then as seen above, $\lambda$ has to be nonnegative valued and we have for some $\alpha$,

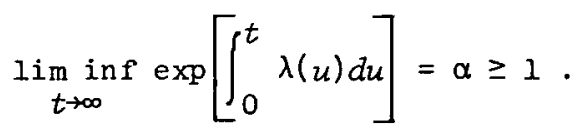

Let $\left\{t_{n}\right\} \rightarrow \infty$ as $n+\infty$ be such that

$$
\alpha=\lim _{n \rightarrow \infty} \exp \left[\int_{0}^{t} \lambda(u) d u\right]
$$

We note from (3.5) that 
(3.9) $\int_{0}^{t} \lambda(u) d u$

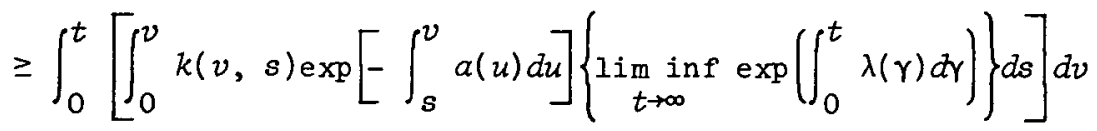

or

$$
\log \alpha_{n} \geq a_{n} \int_{0}^{t}\left\{\int_{0}^{v} k(v, s) \exp \left[-\int_{s}^{v} a(u) d u\right] d s\right\} d v
$$

where

$$
\alpha_{n}=\exp \left[\int_{0}^{t} \lambda(\gamma) d \gamma\right]
$$

Thus

$$
\frac{\log \alpha_{n}}{\alpha_{n}} \geq \int_{0}^{t} n\left\{\int_{0}^{v} k(v, s) \exp \left[-\int_{s}^{v} a(u) d u\right] d s\right\} d v
$$

If $\alpha$ is finite then we have from (3.11) that

$$
\lim _{n \rightarrow \infty} \frac{\log \alpha_{n}}{\alpha_{n}} \geq \underset{t \rightarrow \infty}{\lim \inf } \int_{0}^{t}\left\{\int_{0}^{v} k(v, s) \exp \left[-\int_{s}^{v} a(u) d u\right] d s\right\} d v
$$

and hence

$$
\frac{1}{e} \geq \lim \inf _{t \rightarrow \infty} \int_{0}^{t}\left\{\int_{0}^{v} k(v, s) \exp \left[-\int_{s}^{v} a(u) d u\right] d s\right\} d v .
$$

If $\alpha$ is not finite, (3.11) leads to

$$
\int_{0}^{t}\left\{\int_{0}^{v} k(v, s) \exp \left[-\int_{s}^{v} a(u) d u\right] d s\right\} d v \leq 0 .
$$

Both (3.13) and (3.14) contradict (3.6) and hence there cannot be real $\lambda$ satisfying (3.5). If however (3.6) fails to hold real $\lambda$ can exist and the proof is complete.

We conclude with a few remarks. Integrodifferential equations can represent as special cases delay-differential or functional differential equations. We have provided a set of a priori verifiable sufficient conditions for the existence of oscillatory solutions for scalar integro- 
differential systems. The result of Corollary $2: 1$ is well known in the literature. (Bellman and Cooke [1], Goel et al [5], Smith [9].) As a special case of Corollary 2.2, one can derive some results of Ladde [7]. It is an open problem to examine sufficient conditions for linear coupled systems to have oscillatory solutions. However results on the asymptotic stability and decay rate of solutions of linear coupled integrodifferential systems are available in a recent article of the author (Gopalsamy [6]).

\section{References}

[1] Richard Bell Iman, Kenneth L. Cooke, Differential-difference equations (Mathematics in Science and Engineering, 6. Academic Press, New York, London, 1963.

[2] Fred Brauer and John A. Nohel, The qualitative theory of ordinary differential equations. An introduction (Benjamin, New York, Ams terdam, 1969.

[3] Ruel V. Churchill, Operational mathematics, second edition (McGrawHill, New York, Toronto, London, 1958).

[4] E. Corduneanu and V. Lakshmikantham, "Equations with unbounded delay: a survey", Nonlin. Anal. 4 (1980), 831-877.

[5] Narendra S. Goel, Samaresh C. Maitra and Elliott W. Montroll, "On the Volterra and other nonlinear models of interacting populations", Rev. Moderm Phys. 43 (1971), 231-276.

[6] K. Gopalsamy, "Stability and decay rates in a class of linear integrodifferential equations", Funkcial Ekvacioj (to appear).

[7] G.S. Ladde, "Stability and oscillations in single-species processes with past memory", Intermat. J. Systems Sci. 10 (1979), 621-647.

[8] Richard K. Miller, Nonlinear Volterra integral equations (Benjamin, Menlo Park, California, 1971). 
[9] J. Maynard Smith, Models in ecology (Cambridge University Press, Cambridge, 1974 ).

School of Mathematics,

Flinders University of South Australia,

Bedford Park,

South Australia 5042,

Australia. 